\title{
Johann Ulrich Bilguer (1720-1796) und die Medizinische Fakultät Halle
}

(Zum 250. Geburtstag des Schweizer Chirurgen)

Von Wolfram Kaiser (Halle-Wittenberg)

Am 7.Juli 1748 notiert Professor Johann Juncker (1679-1759) als amtierender Dekan der Medizinischen Fakultät Halle im Geschäftstagebuch, ein in Schlesien stationierter Militärwundarzt sei über die Behandlung eines Kavalleristen beraten worden: «hat der Regiments Chirurgus Herr Bilguer aus Neustadt im Fürstenthum Oppeln ein consilium medicum für einen Reiter, der ulcere vesicae urinariae laboriret, eingeholet ${ }^{19}$. Achtzehn Monate später findet sich eine ähnliche Eintragung; der nunmehr als Dekan fungierende Andreas Elias Büchner (1701-1769) - er hat ein medizinisches und ein philosophisches Doppelordinariat inne und ist außerdem Präsident der Kaiserlichen Akademie der Naturforscher - vermerkt am 19. Januar 1750: «ist dem Regiments-Feldscherer Bilguer, zu Neustadt in Ober-Schlesien, ein Consilium medicum, für einen Cavalier, so febre lenta cum ruderibus luis venereae laboriret, ausgefertigt worden ${ }^{20}$.

Mit diesen Consilia medica beginnt der wissenschaftliche Kontakt des aus Chur gebürtigen Wundarztes Johann Ulrich Bilguer (1720-1796) zur Academia Fridericiana Halensis, die ihm zehn Jahre später den Doktortitel verleihen wird.

Die Frage liegt nahe, warum sich der damals knapp dreißig Jahre alte Militärchirurg, dessen medizinischer Werdegang über Basel, Straßburg, Paris, Stuttgart -Tübingen und das Collegium medico-chirurgicum in Berlin ging, ausgerechnet an die Medizinische Fakultät in Halle wendet, zu der er bis dahin keinerlei persönliche Beziehungen hatte. Eine erklärende Aussage von Bilguer selbst ist nicht vorhanden, so daß der Nachbetrachter hier nur Vermutungen äußern kann. Vielleicht war es die Tatsache, daß die damals in ihrer höchsten Blüte stehende und meistbesuchte deutsche Universität sich aus diesem Grunde geradezu anbot. Nicht ausgeschlossen wäre aber auch, daß Bilguer zunächst ohne eigenes Zutun zu dieser Kontaktnahme kam; die halleschen Fakultätsakten enthalten nämlich eine 
Eintragung des Ordinarius Michael Alberti (1682-1757) vom 12.0ktober 1747 über «ein responsum zur Justificirung eines Regiments Feldscherers zu Neustadt in Ober-Schlesien in puncto curatae Sarcocele ${ }^{18}$. Diese nicht näher explizierte Formulierung wäre auf einen Vorgang beziehbar, bei welchem eine Anfrage über die Therapiemaßnahmen (des namentlich nicht genannten) Wundarztes nach Halle ging, zu seiner «Justificirung» führte und Anlaß zu seiterer medizinischer Korrespondenz wurde.

Bilguer nennt später in seinem von ihm selbst niedergeschriebenen Curriculum vitae eine Reihe von Persönlichkeiten, die auf seine Entwicklung Einfluß nahmen und deren Ausbildung an der Universität Halle erfolgte vielleicht liegen hier zusätzliche Gesichtspunkte, die ihn veranlaßten, sich 1748 und 1750 gerade nach Halle zu wenden.

Dieser Personenkreis beginnt bereits mit dem bei H.MüLLER ${ }^{12}$ genannten Paten des späteren Chirurgen, dem Obristen M. v. Salis: ein Hieronymus v. Salis hatte 1716 als Jurastudent in Halle geweilt (Immatr.: 13. März 1716). Unter seinen Lehrern am Gymnasium der Heimatstadt nennt Bilguer die Professoren Mayer und Zaff: ein J.G. Mayer war 1704 in Halle gewesen, ein Nikolaus Zaff aus Chur hatte 1723 in der Saalestadt studiert (Immatr.: 9. Oktober 1723). Später werden ihm während seines medizinischen Ausbildungsganges weitere einstige "Hallenser» begegnen: vermutlich war Bilguer also schon damals genau über die Situation an der Academia Fridericiana informiert.

Als die eingangs genannten Consilia medica von Halle aus nach Neustadt erteilt werden, steht Bilguer erst seit wenigen Jahren in preußischen Diensten, in die er mehr oder minder zufällig trat. Denn eigentlich hatte der am 1. Mai 1720 in Chur als Sohn des Meisters Johann Luzius Bilguer und seiner Ehefrau Anna Gampser geborene Johann Ulrich Bilguer seine militärmedizinische Laufbahn 1741 in Württemberg begonnen - und auch diese Verpflichtung war wohl nicht von vornherein beabsichtigt gewesen.

Als Geburtsdatum Bilguers wurde von Müller im Kirchenbuch der St.-Martins-Kirche von Chur der 21. April 1720 ermittelt $^{12}$. Diese Differenz zum "offiziellen Geburtsdatum» könnte evtl. darauf zurückzuführen sein, daß im Jahre 1720 in der im Glauben deutlich gespaltenen Stadt die Protestanten den Gregorianischen Kalender vielleicht noch nicht eingeführt hatten ${ }^{13}$.

Folgt man den Daten des Bilguerschen Curriculum vitae, so beginnt seine medizinische Laufbahn im Jahre 1737 mit der Aufnahme des Studiums in Basel, wo er sich an Johann Rudolph Zwinger (1692-1777) anschließt, den Sohn des 1724, verstorbenen Theodor Zwinger (1658-1724).

An den Chirurgen Vaquin nach Straßburg empfohlen, wendet er sich 1738 nach dort und hört hier u.a. auch die Kollegs von Johann Salzmann (1672-1738), Georg Heinrich Eisenmann (1693-1768), Johann Philipp 
Gravel (1711-1761), Franz Balthasar von Lindern (1682-1755) und Johann Jakob Fried (1689-1769). Insbesondere die chirurgisch-ärztliche Kunst von Vaquin ist es, zu der er sich hingezogen fühlt und die nun auch sein Hauptaufgabengebiet wird. Auf Anraten des Lehrers holt er sich Testate über seine schon vor der Straßburger Zeit abgeleistete chirurgische Tätigkeit ein und wird daraufhin Mitglied der Societas Chirurgorum Argentoratensium, also in die hochangesehene Zunft aufgenommen. In Paris sucht er dann seine Kenntnisse am Hôtel Dieu und an der Charité zu vervollständigen - und hier tritt nun der Zufall ein, der seine weitere Laufbahn bestimmt: die dort weilende Herzogin von Württemberg - die Witwe des Feldmarschalls Carl Alexander von Württemberg, eine Prinzessin von Thurn und Taxis - verpflichtet ihn als Militärwundarzt in ihr Heimatland, das sich damals einigen schwerwiegenden politischen Pressionen gegenübersieht.

Württemberg hatte sich unter Carl Alexander infolge ständiger Konflikte mit Frankreich eine starke Armee aufzubauen gesucht. Wenige Jahre nach dem Tode des Herzogs waren der erste Schlesische Krieg und der Bayrische Erbfolgekrieg ausgebrochen. Württemberg sucht Anschluß an Preußen - ein den politischen Plänen Friedrichs II. entgegenkommendes Vorhaben. Die württembergischen Landstände beschließen 1741, die Kinder des verstorbenen Herzogs nach Berlin zu schicken, um sie vor durchziehenden französischen Truppen in Sicherheit zu wissen. Der knapp vierzehnjährige Prinz Carl Eugen - er begründet später die Hohe Carls-Schule - reist damals über Bayreuth an den preußischen Königshof; nach seiner Großjährigkeitserklärung schließt er einen Vertrag mit Friedrich II., welcher letzterem Truppenanwerbungen in Württemberg gestattet. Später heiratet Carl Eugen eine Nichte Friedrichs II. aus der Schwedter Linie.

Das ist der politische Hintergrund der Situation in Württemberg, als Bilguer 1741 nach Stuttgart kommt. Hier und an der Universität Tübingen setzt er seine Ausbildung fort; er hört bei Burkhard David Mauchart (1696-1751) und bei Daniel Hoffmann (1695-1752) und absolviert den Cursus medico-chirurgicus. Zum Chirurgenmajor ernannt, heiratet er 1742 in Heslach Johanne Friederike Mögling, die Tochter eines Berufskollegen ${ }^{22}$.

Dann wird sein Regiment 1742 nach Berlin überstellt: die preußische Karriere Bilguers beginnt. Auch jetzt bemüht er sich um Erweiterung seiner Kenntnisse, als er an der Charité bei Samuel Schaarschmid (1709-1747), Johann Theodor Eller (1689-1760) und bei Johann Georg Lesser Kollegs besucht.

Schaarschmid ist der Sohn eines halleschen, längere Zeit in Rußland tätigen Theologen. Er hatte in der Saalstadt studiert (Immatr.: 20. Februar 1725) und hier 1729 sowie 1736 disputiert. Als Garnisonsarzt und Professor ans Theatrum anatomicum Berolinense ge- 
wählt, gilt seine Aufmerksamkeit auch der Militärmedizin; seine "Abhandlung von Feldkranckheiten » kommt allerdings erst posthum im Jahre 1758 heraus. Auch der Feldmedikus und spätere Berliner Stadtphysikus Johann Georg Lesser ist hallescher Doktorand. Seine 1735 unter Friedrich Hoffmann (1660-1742) vorgetragene Dissertation «de militum valetudine tuenda in castris» ist militärmedizinischen Inhalts.

Schließlich war auch Johann Theodor Eller vorübergehend als Student in Halle gewesen (Immatr.: 27. Januar 1729), bevor er sein Amt an der Charité antrat.

Bilguer nimmt als Infanterie-Feldmedikus an der Schlacht von Kesselsdorf teil und wechselt nach Friedensschluß zum Geslerschen Dragonerregiment über. Es ist im Raum Oppeln stationiert, als die Consilia medica von 1748 und 1750 den direkten Kontakt zwischen Bilguer und den Vertretern der Medizinischen Fakultät Halle - Johann Juncker und Andreas Elias Büchner - herstellen. Letzterem Ordinarius wird er dann 1762 sein Curriculum vitae zustellen, das über diese Zeitspanne zunächst folgendes berichtet $^{1}$ :

«Ego Joannes Ulricus Bilguerus, Curia Rhaetus, natus Sum Anno hujus Seculi 1720 1. Mayas Patre Joanne Lucio, Tribuno Plebis. Qui cum in me non minimam Aviditatem discendi animadverteret, me Doctrinarum Studiis expoliendum tradidit, in Disciplinam Doctissimorum virorum et Professorum Mayer et Zaff qui Gymnasium illustre, quod in urbe patria floret, tunc temporis ornabant. Illorum virorum Disciplina ad annum usque aetatis Decimum Septimum, Seculi tricesimum Septimum ita usus Sum, ut eodem anno ad Basilenses dimitterer, artem medicam discendi causa. Quo quidem consilio commendatus praecipue viro celeberrimo Zwingerto medicinae Doctore, hujus hospitio, convictu, institutione denique usus fui per annum. Quo tempore factum est, ut viro perillustri Bernardoni, Galliae Regis ad Rhaetos tunc Legato innotescerer, ab eodemque adamatus commendarer Vaquino, viro rerum Suarum admodum perito, et Anatomiae Demonstratore Regio, in Nosocomio gallico, quod a Galliae Rege in urbe Argentorati in incrementum Anatomiae et Chirurgiae Sapienti consilio constitutum est. Petii igitur Argentoratum 1738. ibidemque Medicinae Studiosorum numero adjectus, totum me contuli ad medicarum et chirurgicarum doctrinarum Magistros, inprimisque, eos, qui tum principes numerabantur, Salzmannum, Eisenmannum, Gravelium, Lindern, Baehrium, Friedium, Marium, Riekium, Vaquinum, a quibus non docendo tantum, sed consulendo etiam juvabar Vaquini hospitio et convictu utens. Ab his praeceptoribus per quatuor Semestria hyberna et tria aestiva omnes artis medicae partes edoctus, et in Valetudinario praecipue ab obeundas operationes chirurgicas, artemque dextre fasciandi discendam admissus fui. Quo quidem effectum est, ut ea artis pars, quae manu medetur, me pene totum ad Se raperet, ejusque tantus amor in me accenderetur, ut Vaquini consiliis cedens litteras testantes, me Chirurgiae operam dedisse, ex patria peterem, easdemque producendo Societati chirurgorum Argentoratensium memet immiscerem, eidemque Socius adjungerer. Post haec Lutetiam Parisiorum profectus, celeberrima per universam Europam nosocomia, tum illud, quod L'hotel Dieu, tum alterum, quod Charité vocatur, nec non alia publica aeque ac privata artis nostrae theatra sedulo frequentavi. Dum Lutetiae commorarer, Serenissima conjux Ducis Wurtembergici Legionem ferentariam conduxerat, cujus Militibus ut tanquam chirurgus major adessem, ego Lutetia Stuttgardiam evocabar, et a celeberrimis tum viris, Maucharto, Hoff- 
manno, Bacmeistero, Medicis, Bauero vero et Simonio Chirurgis Tubingae examinatus, cursumque, quem vocant, medicum et chirurgicum, coram ipsis emensus, Legioni isti anno 1741. dabar chirurgus Major. Sed cum Legio ista anno 1742. potentissimo Borussorum Regi traderetur, ejusque exercitibus concorporaretur, ego quoque una cum tota Legione Berolinum ingressus, artis peritam qualemcunque viris Illustribus, Ellero, Schaarschmidio et Lesserto comprobavi, Spartam, quam nactus eram, in posterum etiam ornare jussus. In bello illo, quod anno 1744. et 45. in Bohemia et Saxonia sparserat, commilitonibus meis opem attuli, ac praecipue post nobilem illam pugnam, prope pagum Kesselsdorff pugnatam, Sauciatos milites omnes meae et Cl. Brebischii Chirurgi majoris Legionis Praetorianae, curae traditos, qua potui, diligentia et dexteritate curavi, laborisque fractum tali non contemnendum. Potentissimi enim Regis clementia memet Legione illa terentaria in Legionem Cataphragtorum militum, cui tum a Praefecto Geslero, nunc a Schmettavio nomen est, transire iussit, multoque adeo largiore stipendio affecit ....

\section{In Übersetzung:}

«Ich, Johann Ulrich Bilguer aus Chur im Rhätischen, bin am 1.Mai 1720 als Sohn des Zunftmeisters Luzi Bilguer geboren. Dieser wandte seine größte Mühe darauf, mich etwas Rechtes lernen zu lassen; er schickte mich in das heimatliche Gymnasium und übergab mich der Aufsicht der Professoren Mayer und Zaff. Bis zu meinem 17.Lebensjahr, also bis 1737, habe ich aus dem Unterricht dieser Männer Nutzen gezogen. Dann wurde ich im gleichen Jahre zur Erlernung der Ars medica nach Basel geschickt. Ein Jahr lang war ich als Hausgenosse und Schüler dem berühmten Arzt Dr. Zwinger anvertraut. Danach wurde ich durch Vermittlung des französischen Legaten Bernardoni an Vaquin empfohlen, einen erfahrenen Mann, der als königlicher Demonstrator für Anatomie am französischen Hospital in Straßburg wirkte, das durch den König von Frankreich in Straßburg in weisem Beschluß zwecks Verbesserung der anatomischen und chirurgischen Ausbildung errichtet worden war. Also ging ich 1738 nach Straßburg und trug mich dort als Medizinstudent ein; ich schloß mich eng an die führenden Vertreter von Medizin und Chirurgie an, nämlich an Salzmann, Eisenmann, Gravel, Lindern, Bach, Fried, Marius, Rieck, Vaquin; ich hörte ihre Kollegs und beobachtete ibre Praxistätigkeit, wobei ich bei Vaquin Wohnung nahm. Vier Sommerund drei Wintersemester hindurch wurde ich von den genannten Lehrern in allen Künsten der Medizin erzogen; im Frankenhaus wurde ich vor allem bei den anstehenden chirurgischen Operationen und zur Erlernung des regelrechten Verbindens herangezogen. Dadurch kam es, daß der manuelle Teil der ärztlichen Kunst fast völlig Besitz von mir ergriff und ich auf Anraten von Vaquin mir schriftlich aus meiner Heimat bescheinigen ließ, dort bereits chirugisch tätig gewesen zu sein, um durch Vorlage einer derartigen Bestätigung Mitglied der Straßburger Chirurgenzunft zu werden. Im Anschluß brach ich nach Paris auf und besuchte dort fleißig die in ganz Europa berühmten Spitäler Hôtel Dieu und Charité wie andere staatliche und private Institutionen.

Während meines Aufenthaltes in Paris veranlaßte dort die Herzogin von Württemberg die Aufstellung einer militärischen Einheit, in die ich als Chirurgenmajor eintrat und dann nach Stuttgart überstellt wurde, wo ich von den weithin berühmten Ärzten Mauchart, Hoffmann, Bacmeister sowie von dem Chirurgen Bauer und Simon in Tübingen examiniert wurde und den Cursus medico-chirugicus ableistete. Der Rang des Chirurgenmajors ver- 
blieb mir. Als 1742 das Regiment an den König von Preußen überstellt wurde, kam ich nach Berlin und wurde hier durch Eller, Schaarschmid und Lesser überprüft; auch jetzt konnte ich meinen militärischen Rang behalten. Im Böhmischen und Sächsischen Feldzug von 1744 und 1745 konnte ich meinen Kameraden ärztliche Hilfe leisten, und besonders nach der Schlacht von Kesselsdorf wurden die mir und meinem Kollegen Brebeschius übergebenen Verwundeten nach bestem Wissen und Können versorgt. Auf königlichen Befehl wechselte ich dann unter guten Bedingungen zum Dragonerregiment Gesler über, das jetzt das Schmettausche heißt.

Im Verband des Schmettauschen Regiments bin ich nach der Schlacht von Prag im Jahre 1757 zum Kommandanten eines stark belegten Lazarettes ernannt worden und bald darauf mit den Verwundeten nach Dresden aufgebrochen, wobei ich nichts versäumt habe, was zum Heile der übel Verwundeten notwendig war. Nach der Schlacht von Roßbach nach Dresden berufen, empfing ich neue Order, verwundete französische Soldaten in preußischer Gefangenschaft ärztlich zu versorgen. Während ich noch hiermit beschäftigt war, wurde in Schlesien die Schlacht bei Leuthen geschlagen; bei der Vielzahl von Verwundeten reichten dort die Lagerchirurgen nicht aus. Deshalb brach ich zwecks Versorgung der ungeheuren Zahl von Verwundeten auf königlichen Befehl zusammen mit 60 Chirurgen von Leipzig nach Beslau auf. Im Dezember dieses Jahres starb der Generalchirurg Bonessius, auf dessen Stelle ich berufen wurde. 1758 habe ich in Liegnitz, Jauer und Striegau Lazarette eingerichtet und geleitet, um die Soldaten zu versorgen, welche bei der Belagerung von Schweidnitz verwundet oder erkrankt waren. Vom März bis zur Schlacht von Kunersdorf diente ich zunächst bei der Armee des Prinzen Heinrich, dann im königlichen Heere selbst; dabei sorgte ich für das Lazarettwesen insgesamt. Nach der Schlacht von Kunersdorf wurde ich von dort auf königliche Order zunächst nach Küstrin und dann nach Stettin geschickt und habe dort alle Dispositionen für ein gut eingerichtetes Lazarett getroffen, und zwar derart, daß ich aus der ungeheuren Anzahl von Verwundeten mit zehn Herzögen im Generalsrang und 40 höheren Offizieren die meisten heilen konnte, worüber ich anderenorts berichtete (siehe meine Inauguraldissertation über Fragen der Amputation). Im Heer des Prinzen Heinrich und dann des Königs nahm ich 1760 an der dreitägigen Novemberschlacht von Torgau teil, und auch hier habe ich für die ungeheure Anzahl von Verwundeten alles Notwendige getan. 1761 habe ich mich um den Doktorgrad bemüht und meine oben erwähnte Arbeit der Medizinischen Fakultät Halle vorgelegt. Im September des gleichen Jahres wurde ich von der Königlichen Sozietät der Wissenschaften in Göttingen als Mitglied aufgenommen.»

Die folgenden Jahre übergeht Bilguer in seinem Curriculum vitae. Es setzt erst wieder mit dem Siebenjährigen Krieg ein, wo Bilguer im Dezember 1757 zum Generalchirurgen ernannt wird. Es ist anzunehmen, daß er im Herbst dieses Jahres zumindest kurz in Halle gewesen ist, nahm er doch an der unweit der Saalestadt stattfindenden Schlacht von Roßbach teil. Dann führen ihn seine Aufgaben wieder auf den schlesischen Kriegsschauplatz sowie nach Pommern und schließlich nach Torgau. Hier trifft er mit Ernst Gottfried Baldinger (1738-1804) zusammen, der 1756 in Halle studiert hatte und nach der in Jena durchgeführten Promotion ebenfalls als Militär- 
arzt in preußischen Diensten steht; beide wird fortan eine lebenslange Freundschaft verbinden ${ }^{3}$.

Bilguers umfangreiche Erfahrungen auf dem Gebiet der Kriegschirurgie finden jetzt ihren Niederschlag in der berühmt gewordenen Schrift über die Amputationsindikation, welche er nunmehr als Inauguraldissertation der Medizinischen Fakultät Halle vorzulegen beschließt. Zu diesem Zweck kommt er persönlich nach Halle und unterwirft sich den üblichen Prüfungen, muß aber auf die regulären Promotionsformalitäten verzichten, da ihn seine militärärztlichen Pflichten ins Heer zurückrufen. Ein Jahr später wird er dann an Andreas Elias Büchner in Halle die folgende Schilderung der Kriegsjahre bis 1761 und der erfolgten Promovierung geben ${ }^{1}$ :

«Prioribus annis hujus, quod nunc geritur, belli Legioni illi Schmettaviae adjunctus post pugnam illam, prope urbem Praguae anni 1757 pugnatam nosocomio militari, non exiguum Sauciatorum numerum continenti praefectus, et paulo post cum iis Dresdam profectus, nihil eorum neglexi, quae ad Sanitatem misere afflictorum pertinerent. Quo quidem mandato munere fungens post pugnam prope pagum Roßbach pugnabam, Dresda evocatus, nova mandata accepi, ut vulneratos milites ex Gallorum exercitu, in Borussorum potestatem redactos, curatione me sublevarem. Quo in opere cum adhuc dum versarer, aliud proelium in Silesia prope pagum Leuthen commissum, tot milites vulneribus afflixerat, ut Chirurgorum castrensium, qui tum in Silesia militibus auxilium offerent, cura non sufficeret. Itaque Potentissimi Regis jussu Lipsia Wratislaviam, qua fieri poterat, diligentia una cum sexaginta Chirurgis castrensi nosocomio adjunctis, iter fecimus, ingentem vulneratorum numerum curaturi. Eodem anno et mense quidem Decembri Cl. et peritissimus Bonessius, qui hactenus officio Chirurgi generalis functus erat, e vita migrabat. In cujus locum me sufficiebat gratia et clementia Potentissimi Regis. Anno 1758 in oppidis, Lignitii, Jauriae et Strigaviae imperata nosocomia instruxi iisdemque praefui, milites curaturus, qui obsidione muniti oppidi Schweidnitzii vulnera acceperant morbosque contraxerant. A mense Martio ad id tempus, quo Potentissimus Rex Borussiae cum Russorum exercitu haud procul a pago Kunersdorff certamen iniit, primo exercitum, cui Serenissimus Princeps Regius Henricus Dux erat, deinceps exercitum, quem Rex ipse ducebat, sequutus, ea quae ad rem nosocomii militaris pertinent, ordinavi et direxi. Eo ipso tempore, quo pugna ea pugnabatur, in ipso pugnae loco una cum Chirurgis nosocomii mei militibus sauciatis auxilium attuli, indeque Regis iussu una cum vulneratis omnibus primo Custrinum postea Stetinum petii, atque in hac urbe illa omnia quae nosocomium militare, bene instruendum, poscere videbatur, disposui atque instruxi: sic quidem ut ex ingenti numero vulneratorum, in quibus decem Duces, quos Generales hodie vocant, et plus quam quadringenti alii militum praefecti numerabantur, plurimos in sanitatem restitui, ea usus felicitate, de qua dixi alibi (vid. Dissertatio nostra inauguralis de membrorum amputatione rarissime administranda aut quasi abroganda). Postea tum exercitum, cui Serenissimus Princeps Regius, Henricus praefectus erat, tum eum, cui Rex ipse praeerat, concomitatus, pugnae tandem prope Torgaviam anno 1760, et mensis quidem Novembris die tertio pugnatae interfui, ingentemque sauciatorum militum numerum nactus, nihil omisi, quod aut ad misere afflictorum salutem recuperandam, aut ad artis incrementum facere posset. Anno 1761. ad gradum Doctoris Medicinae et Chirurgiae adspiravi, eoque nomine dissertationem paulo supra excitatam Gra- 
tiosae Facultati, quae in Academia Fridericiana, quae Halae est, floret, examinandam, qua decebat, modestia exhibui. Eodemque anno, et mense quidem Septembri a Regia Scientiarum Societate, quae Goettingae est, in Sociorum numerum cooptatus Sum, litterarumque commercio adjunctus.»

Aus den Fakultätsakten läßt sich der zeitliche Ablauf der Promotionsformalitäten genau verfolgen. Am 13. März 1761 trägt sich «Johannes Huldricus Bilguerus Curia Rhetus Chirurgus Generalis Copiarum Borussicarum» in das Matrikelalbum ein, wobei er die Gebühren in voller Höhe hinterlegt (Matr.-Nr.150/1761). Während des auf wenige Tage befristeten Aufenthaltes legt er seine fertiggestellte Abhandlung vor, unterzieht sich den üblichen Examina und wird nach deren Bestehen in einer privatim einberufenen Fakultätssitzung zum Dr. med. et chir. ernannt; die öffentliche Verteidigung der von der Fakultät als Inauguraldissertation anerkannten Arbeit wird ihm erlassen. Im Anschluß leistet er den Ärzteeid. Er hat die Saalestadt bereits wieder verlassen, als Andreas Elias Büchner am 21.März 1761 im Fakultätsalbum vermerkt ${ }^{16}$ : «Praeviis consuetis Examinibus, et praestito ordinario Juramento, in censessu Facultatis privatim in Doctorem Medicinae et Chirurgiae renunciatus fuit Dn.Joannes Ulricus Bilguer, CuriaRhaetus, Generalis Praefectus Chirurgorum Exercitus Regii Borussici, insimulque concessionem obtinuit, ut, quoniam ob officii rationem diu ipsi hic commorari non licuit, loco Dissertationis inauguralis e cathedra publice defendendae, oblatum suum Specimen, de Membrorum amputatione rarissime administranda, aut quasi abroganda, typis exscriptum publici iuris faceret, id quod etiam factum est, obtinuitque Diploma, me, Decano, Promotore.»

Es ist weitgehend bekannt und bedarf an dieser Stelle kaum der erneuten Betonung, daß die Bilguersche Schrift über die Amputationsindikation und vor allem -kontraindikation schnell weltweites Aufsehen erregt, einen Wendepunkt in der Kriegschirurgie darstellt und dem Verfasser den Beinamen «Vater der Konservativen Chirurgie» einbringt ${ }^{11}$. Bilguer selbst besorgt noch im gleichen Jahr eine deutsche Ausgabe; Übersetzungen ins Französische (1764), Englische (1764), Holländische (1771) und Spanische (1782) folgen.

Sieben Monate nach der Promotion von Bilguer wird einem zweiten Schweizer Militärchirurgen von Halle aus die gleiche Ehrung zuteil: Johann Heinrich Roth aus Keßwil, Wundarzt in französischen Diensten, erhält am 26. Oktober 1761 in abesentia den Doktortitel zugesprochen. Hierüber heißt es im Fakultätstagebuch ${ }^{17}$ : "Dominus Johannes Henricus Roth, Keswila-Turgoviensis, Helvetus, Cohortis Schoenbergianae in Regis Gallico exercitu Chirurgus Supremus, ob egregiam in variis Academicis olim acquisitam, et ordini nostrae 
specimine quodam, de dysenteria, docte elaborato, sufficienter demonstratam scientiam, absens obtinuit Diploma doctoris Medicinae et Chirurgiae.»

Schließlich wird dreißig Jahre später noch ein dritter Schweizer Chirurg von der Medizinischen Fakultät der Academia Fridericiana promoviert werden: im November 1791 stellt man hier das Doktordiplom für den aus Rorschach gebürtigen Franz Leopold Lafontaine (1756-1812) aus, welcher damals als Leibmedicus in Polen tätig ist.

Von Halle aus begibt sich Bilguer zur Armee des Prinzen Heinrich zurück, die nach der Schlacht von Torgau (3. November 1760) im Sächsischen stationiert war. Hier stellt er die Pars prima seiner «Chirurgiae Institutiones» zusammen; das Buch erscheint 1762 in Glogau als «Anweisung zur ausübenden Wund-Artzeney in Feld-Lazarets». Mit seinem Freund und Kollegen Baldinger, dem die Heeresleitung die Fortsetzung seiner Studien in Wittenberg gestattete, bleibt er dabei in ständigem Kontakt. Als dieser am 18.Januar 1763 dort die Inauguraldissertation «de militum morbis» vortragen läßt ${ }^{2}$, kann er in der gedruckten Arbeit bereits auf die weiteren Pläne Bilguers hinweisen: «Continebit pars altera et tertia singulares singularum partium laesiones, feliciter persanatas.» Zur halleschen Doktorarbeit Bilguers notiert er an gleicher Stelle: «Longo rerum usu edoctus, cognovit Clariss. Auctor, membra integra saepius conservari posse, ac vulgo creditur.» Begeisterter Anhänger Bilguers wird auch Ulrich Christoph Salchow (1722 bis 1787), hallescher Doktorand von 1746 und nunmehr Physikus in Dithmarschen; er bezeichnet Bilguer als den «größten Wundarzt seiner Zeit. ${ }^{15}$

Auch der Kontakt zur Medizinischen Fakultät Halle ist zu diesem Zeitpunkt für Bilguer nicht abgerissen: Andreas Elias Büchner nimmt Bilguer am 17. Mai 1762 - knapp zwei Wochen vorher haben Preußen und Rußland den Kriegszustand beendet - als 649. Mitglied in die Academia Imperialis Leopoldino-Carolina Naturae Curiosorum auf (Cognomen: Nymphodorus IV.). Bei dieser Gelegenheit stellt Bilguer dem Akademiepräsidenten das zitierte Curriculum vitae $\mathrm{zu}^{1}$.

Wenige Wochen nach dem Bilguerschen Halle-Besuch war im April/Mai $1761 \mathrm{mit}$ Leonhard Euler (1707-1783) ein prominenter Landsmann in der Saalestadt gewesen ${ }^{7}$ : er hatte seinen Sohn Karl Euler (1740-1790) begleitet, der hier sein Medizinstudium abschließen sollte (Dr. med. am 2.Oktober 1762). Gewohnt hatte Euler bei dem ihm wohlbekannten Physiker und Mathematiker Johann Andreas v. Segner (1704-1777), der hier seit 1755 lehrte.

Johann Ulrich Bilguer ist der fünfte Schweizer, dem während der halleschen Amtszeit von Andreas Elias Büchner (1745-1769) die Ehre einer Mitgliedschaft in der «Leopoldina» zuteil wird. Vorher hatte Büchner Johann Gesner (am 4.September 1746), Albrecht von Haller (am 10. Januar 
1750), Franz Rudolph von Schachheim (am 3. März 1759) und den Schaffhauser Lorenz Spengler (am 12.0ktober 1761) in diese renommierte Gesellschaft berufen. Nach Bilguer werden dann noch Johann Caspar Sulzer, Karl Bonnet aus Genf (am 10. Januar 1763) und der Berner Naturgeschichtler Theophil Sigismund Gruner (am 12. Juni 1766) folgen.

Der weitere Lebensweg des nunmehr vorwiegend in Berlin tätigen Johann Ulrich Bilguer sowie sein wissenschaftliches Werk ${ }^{14,21}$ stehen an dieser Stelle nicht zur Abhandlung an, zumal hierüber wiederholt berichtet wurde $^{3-6,8-12,21}$. Zweifellos ist es später stiller um Bilguer geworden, der 1786 nicht auf die Stelle des verstorbenen ersten Generalchirurgen Johann Leberecht Schmucker (1720-1786) aufrücken kann; er muß zusehen, wie Johann Christian Anton Theden (1714-1797) nachrückt. Über die Entwicklung in Halle ist er jedenfalls auch damals noch sehr gut informiert gewesen; das geht aus einem jüngst edierten Schreiben aus dem Jahre 1788 hervor, in welchem er seinem Bruder Daniel nach Chur Mitteilung über die halleschen Schulverhältnisse macht ${ }^{12}$ : «Das Paedagogium regium in Halle wird sehr gerühmt seit der Professor Niemayer Zoologie hält in gedruckter Anweisung, worin die Lehrstunden und alle Disziplinen genau bestimmt.»

Johann Ulrich Bilguer ist am 6.April 1796 in Berlin gestorben. Die 250. Wiederkehr seines Geburtstages - am 1. Mai 1970 - gibt Anlaß, insbesondere auch von der Stätte seiner wissenschaftlichen Graduierungen aus des Wirkens des berühmten Kriegschirurgen zu gedenken.

\section{Literatur}

1. Archiv der Deutschen Akademie der Naturforscher (Leopoldina); Briefarchiv Nr.649

2. Baldinger E.G., De millitum morbis inprimis vero exercitus Regis Prussiae, Resp. A. I. Bose, Wittenberg 1763

3. Baldinger E.G., Biographien jetzt lebender Ärzte und Naturforscher in und außer Deutschland, Göttingen 1770

4. Buess H., Johann Ulrich Bilger, Praxis 19 (1946) 292

5. Farner C., Der Bündner Chirurg Johann Ulrich Bilguer und sein Werk über die Hypochondrie, Bündner Monatsblatt 1963, S. 281

6. HeINsinus TH., Denkwürdigkeiten nach Tagsgeschichten der Mark Brandenburg, Berlin 1796

7. JušKevič A.P., Leonhard Euler und die Universität Halle, Nova Acta Leopoldina N. F. 27 (1963) 367

8. KaISer W. und H.Krosch, Hallesche Doktoranden als Mitglieder der Academia Imperialis Leopoldino-Carolina Naturae Curiosorum, Wiss. Z. Univerität Halle XVI (1967) 603 
9. KöHLER A., Die Kriegschirurgen und Feldärzte Preußens und anderer deutscher Staaten in Zeit- und Lebensbildern, Berlin 1899

10. Meusel J.G., Lexikon der vom Jahr 1750 bis 1800 verstorbenen Teutschen Schriftsteller, Band 1, Leipzig 1802

11. MüLLer H., Über den Churer Kriegschirugen Johann Ulrich Bilguer, Bündner Jahrbuch 68 (1967) 74

12. Müller H., Johann Ulrich Bilg(u)er (geb. 1720 in Chur, gest. 1796 in Berlin), Gesnerus 25 (1968) 116

13. Müller H., Persönliche Mitteilung vom 20. März 1969

14. Nigst H., Johann Ulrich Bilgers physikalisch-mechanische Theorie der Contrafissuren, Schweiz. med. Wschr. 77 (1947) 758

15. Salchow U. Chr., Chirurgische Beobachtungen, Leipzig 1769

16. Univ.-Archiv Halle, Rep. 29 F VII, Nr. 1, p. 46

17. ebendort, p. 47

18. ebendort, p. 162

19. ebendort, p. 163

20. ebendort, p. 164

21. Vogeler K., Johann Ulrich Bilguer und der Streit um die Amputation im 18.Jahrhundert, Münchner med. Wschr. 16 (1928) 750

22. Watzka M., Johann Ulrich v. Bilguer, Neue Deutsche Biographie, Band 2 (1955) S. 236, Berlin 1955 